\title{
Dynamic contrast-enhanced MRI serves as a predictor of HIFU treatment outcome for uterine fibroids with hyperintensity in T2-weighted images
}

\author{
WEN-PENG ZHAO ${ }^{1,2}$, JIN-YUN CHEN ${ }^{1}$ and WEN-ZHI CHEN ${ }^{1,3}$ \\ ${ }^{1}$ State Key Laboratory of Ultrasound Engineering in Medicine Co-founded by Chongqing and the Ministry of Science and \\ Technology, Chongqing Key Laboratory of Ultrasound in Medicine and Engineering, College of Biomedical Engineering, \\ Chongqing Medical University, Chongqing 400016; ${ }^{2}$ Department of Interventional Ultrasound, Chinese \\ People's Liberation Army General Hospital, Beijing 100853; ${ }^{3}$ Clinical Center for Tumor Therapy, \\ Second Affiliated Hospital, Chongqing Medical University, Chongqing 400010, P.R. China
}

Received July 17, 2014; Accepted July 2, 2015

DOI: 10.3892/etm.2015.2879

\begin{abstract}
The aim of the present study was to investigate the efficacy of dynamic contrast-enhanced magnetic resonance imaging $(\mathrm{MRI})$ in predicting the outcome of using ultrasound-guided high-intensity focused ultrasound (USgHIFU) ablation for the treatment of uterine fibroids with T2 hyperintensity under MRI. A total of 131 uterine fibroids from 131 patients that appeared hyperintense under T2-weighted MRI were analyzed. The uterine fibroids were subjectively categorized into slight, irregular or regular enhancement groups, according to pretreatment dynamic contrast-enhanced MRI in the arterial phase within $60 \mathrm{sec}$ after the injection of gadolinium. The non-perfused volume (NPV), which is indicative of successful ablation, was represented as the non-perfused area inside the uterine fibroids on enhanced MRI scans following treatment. Additionally, the treatment duration, treatment efficiency, sonication duration, energy efficiency ratio and any adverse events were recorded. The results indicated that the average NPV ratio for all the treated fibroids was $68.5 \%$, while the average NPV ratios for fibroids with slight, irregular or regular enhancement were 84.7, 70.6 and $57.1 \%$, respectively. Fibroids with regular enhancement were associated with the lowest NPV ratio and the lowest treatment efficiency, but exhibited the highest energy effect ratio and an elevated risk of severe adverse effects. The results of the present study indicate that hyperintense uterine fibroids with slight and irregular enhancement in the arterial phase of dynamic
\end{abstract}

Correspondence to: Professor Wen-Zhi Chen, Clinical Center for Tumor Therapy, Second Affiliated Hospital, Chongqing Medical University, 74 Linjiang Road, Chongqing 400010, P.R. China E-mail: chenwz@haifu.com.cn

Key words: dynamic contrast-enhanced magnetic resonance imaging, ultrasound-guided high-intensity focused ultrasound, hyperintense uterine fibroids contrast-enhanced MRI are suitable for USgHIFU treatment. By contrast, uterine fibroids with regular enhancement were associated with the lowest treatment efficacy and safety.

\section{Introduction}

High-intensity focused ultrasound (HIFU) may be used to accurately and visually ablate target tissue. It has been used in clinical practice worldwide to ablate uterine fibroids, and has been proposed as a safe, feasible $(1,2)$ and cost-effective (3) alternative for the treatment of uterine fibroids.

HIFU is an effective treatment for uterine fibroids and sustained symptomatic relief has been achieved in the majority of patients with symptomatic fibroids (2); however, certain patients have exhibited lower regression rates at follow-up after the initial treatment and an increase in fibroid volume (4). Previous studies $(2,5,6)$ demonstrated that these results may have been due to the reduced non-perfused volume (NPV) observed immediately after HIFU. The extent of the NPV inside the uterine fibroids has been observed using enhanced magnetic resonance imaging (MRI) following treatment, as an indication of treatment outcome. A larger NPV may be associated with enhanced symptom relief and a reduction in fibroid volume $(2,7)$. Fibroids are defined as hyperintense if their pretreatment signal intensity appears to be equal to or greater than the surrounding myometrium under T2-weighted MRI. Previous studies $(8,9)$ have observed that hyperintense fibroids exhibit a smaller average NPV and lower regression rates compared with isointense or hypointense fibroids following HIFU ablation. Therefore, hyperintense fibroids should be exempted from this procedure. However, Gorny et al (10) suggested that the differences in NPV and symptom relief between hyperintense, isointense and hypointense fibroids were not statistically significant. Furthermore, a previous case report suggested that hyperintense fibroids that exhibit delayed enhancement under dynamic contrast-enhanced MRI may be treated successfully using focused ultrasound (11).

The contradicting results of previous studies may be explained by the differences in blood supply among the three 
types of fibroid. Abundant blood supply may prevent ultrasound energy deposition and diminish heat sink in uterine fibroids, thereby affecting ablation outcomes. Yamashita et al (12) observed that degenerated and cellular uterine fibroids manifested as hyperintense under T2-weighted MRI. There was considerable overlap, and dynamic contrast-enhanced MRI was able to reflect the blood flow of uterine fibroids and distinguish between them.

Therefore, the capacity of the dynamic contrast-enhanced MRI characteristics of hyperintense uterine fibroids to predict the clinical outcomes of HIFU ablation remains unclear. The aims of the present study were to investigate the role of dynamic contrast-enhanced MRI in predicting the treatment outcome of HIFU ablation used for the treatment of uterine fibroids that appear hyperintense under T2-weighted MRI and to determine the technical success of this method.

\section{Materials and methods}

Clinical protocol. This retrospective study was approved by the Ethics Committee at Chongqing Medical University (Chongqing, China). A total of 131 patients with 131 symptomatic uterine fibroids that appeared hyperintense under pretreatment T2-weighted MRI were analyzed between October 2010 and January 2013 at the First Affiliated Hospital of Chongqing Medical University. Uterine fibroids were defined as hyperintense when their pretreatment signal intensity was equal to or greater than the surrounding myometrium under T2-weighted MRI (Fig. 1) (9). All patients that agreed to participate in this study signed an informed consent form.

The inclusion criteria were as follows: i) Final diagnosis of symptomatic uterine fibroids based on MRI findings; ii) patient age, $>18$ years; iii) premenopausal status; iv) a maximum targeted fibroid diameter of $\geq 2 \mathrm{~cm}$, but $\leq 10 \mathrm{~cm}$; and v) a clear and safe acoustic pathway, with no implants/obstructions in the intestinal canal and acoustic pathway. In addition, the patients were able to clearly communicate with the nurse or physician during the procedure. All the patients agreed to undergo pretreatment and post-treatment (within one month) conventional and dynamic contrast-enhanced MRI scans. The exclusion criteria were as follows: Pregnancy, contraindications for MRI or gadolinium injection solution, a suspected or confirmed malignant uterine tumor, pelvic acute inflammatory disease or uncontrolled systemic disease, inability to remain in a prone position for $3 \mathrm{~h}$, or indications of fibroids with evident spontaneous non-perfused areas on enhanced MRI scans. Furthermore, all patients presented with 1-3 uterine fibroid tumors, and only the dominant fibroid that produced clinically relevant symptoms was treated.

Pre-treatment MRI. Axial breath-hold T1-weighted images [repetition time/echo time (TR/TE), 270/2.1; flip angle, $15^{\circ}$; slice thickness, $8 \mathrm{~mm}$; matrix size, 180x384 mm], multi-section T2-weighted fast spin-echo images (TR/TE, 3,600/105; slice thickness, 6-8 mm; matrix size, 224x320 mm) and dynamic contrast-enhanced T1-weighted gradient-echo images (TR/TE, 4.5/2.0; slice thickness, $0.5 \mathrm{~mm}$; matrix size, $256 \times 288 \mathrm{~mm}$ ) were obtained from all the patients using a Signa Excite-II 1.5T MR unit (GE Healthcare Bio-sciences, Pittsburgh, PA, USA) a number of days prior to treatment and within one month following treatment. Dynamic contrast-enhanced fat-saturated T1-weighted images in axial planes (TR/TE, 3.8/1.8; flip angle, $15^{\circ}$; slice thickness, $2 \mathrm{~mm}$; matrix size, 256x288 mm) were acquired every $20 \mathrm{sec}$ for a duration of $120 \mathrm{sec}$ following the injection of gadolinium $\left(\mathrm{Gd}^{3+} ; 0.1 \mathrm{mmol} / \mathrm{kg}\right.$; Omniscan GE Healthcare Life Sciences, Chalfont, UK).

According to a previous study, the arterial vessels within uterine fibroids are visible within 10-30 sec after the injection of gadolinium, followed by the myometrial vessels, and the arterial vessels disappear after 30-60 sec (13). Therefore, the uterine fibroids in the present study were classified as exhibiting slight, irregular or regular enhancement relative to the myometrium based on dynamic contrast-enhanced MRI within $60 \mathrm{sec}$ after the injection of gadolinium. Slight enhancement was observed when the enhancement degree of the fibroid was reduced compared with that of the myometrium. Regular enhancement was observed when the distribution of the enhanced signal was regular and the enhancement degree was equal to or greater than that of the myometrium. Irregular enhancement was observed when the distribution of the enhanced signal was irregular and the slightly enhanced signal was interspersed among the enhanced signal (Fig. 2).

Ultrasound-guided HIFU (USgHIFU) treatment using a USgHIFU ablation system. The procedure was performed using a JC HIFU Tumor Therapeutic System (Chongqing Haifu Medical Technology, Co., Ltd., Chongqing, China), which provided real-time visual B-mode ultrasound during treatment. A transducer with a diameter of $20 \mathrm{~cm}$ was used at a frequency of $0.8 \mathrm{MHz}$ to produce the required energy, and the focal length was $15 \mathrm{~cm}$. The transducer was fixed in a sac filled with degassed water, and its movement was controlled by a computer (HIFU-C20A; Advantech (China) Co., Ltd., Taipei, Taiwan). The transducer was able to move freely in six directions: Upward, downward, forward, backward, left or right.

Treatment. All the patients underwent careful bowel and skin preparation prior to treatment, which included a diet of liquid food for three days prior to treatment, $12 \mathrm{~h}$ of fasting prior to treatment and an enema $2 \mathrm{~h}$ before the procedure. In addition, hair was shaved on the anterior abdominal wall from the umbilicus to the symphysis pubis, and the skin of the anterior abdominal wall was degreased using $75 \%$ alcohol and degassed using negative pressure suction.

The therapy was conducted while the patients received an intravenous sedative and an analgesic (0.8-1.0 $\mu \mathrm{g} / \mathrm{kg}$ fentanyl; $0.02-0.03 \mathrm{mg} / \mathrm{kg}$ midazolam hydrochloride). Patients remained conscious, and parameters including temperature, respiration, heart rate and blood pressure were monitored during the procedure.

The patients laid in a prone position on the HIFU treatment machine, and the anterior abdominal wall was in contact with the transducer, which was in a sealed sac filled with degassed water. Real-time ultrasound imaging was used to observe the target area and the adjacent structures, the gray-scale changes in the target area and the adjacent structures, including the bowel lumen, bladder and endometrium. A focal point was established from the deep margin to the near margin of the fibroid, $\geq 1 \mathrm{~cm}$ from the boundary of the 

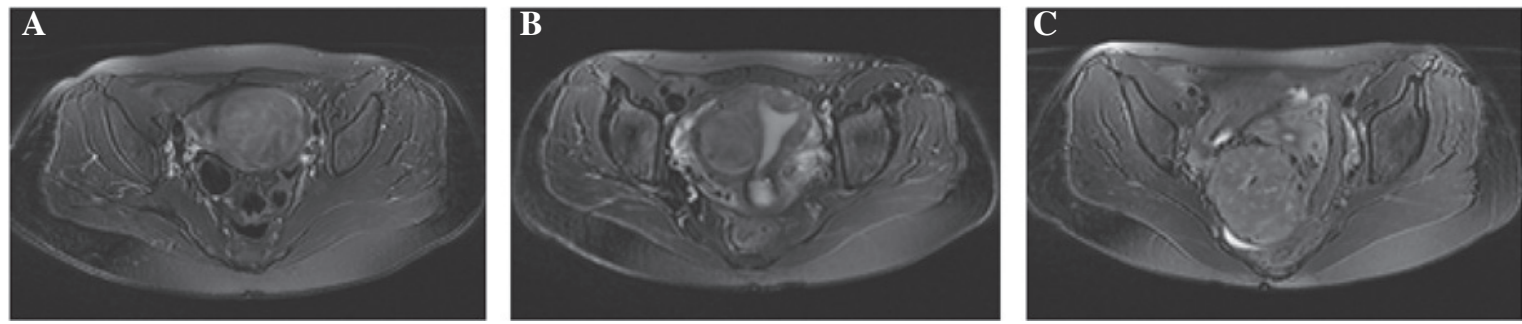

Figure 1. Axial T2-weighted images of patients with hyperintense uterine fibroids. (A) Signal intensity of uterine fibroid is higher than myometrium. (B and C) Signal intensity of uterine fibroids is almost equal to myometrium.
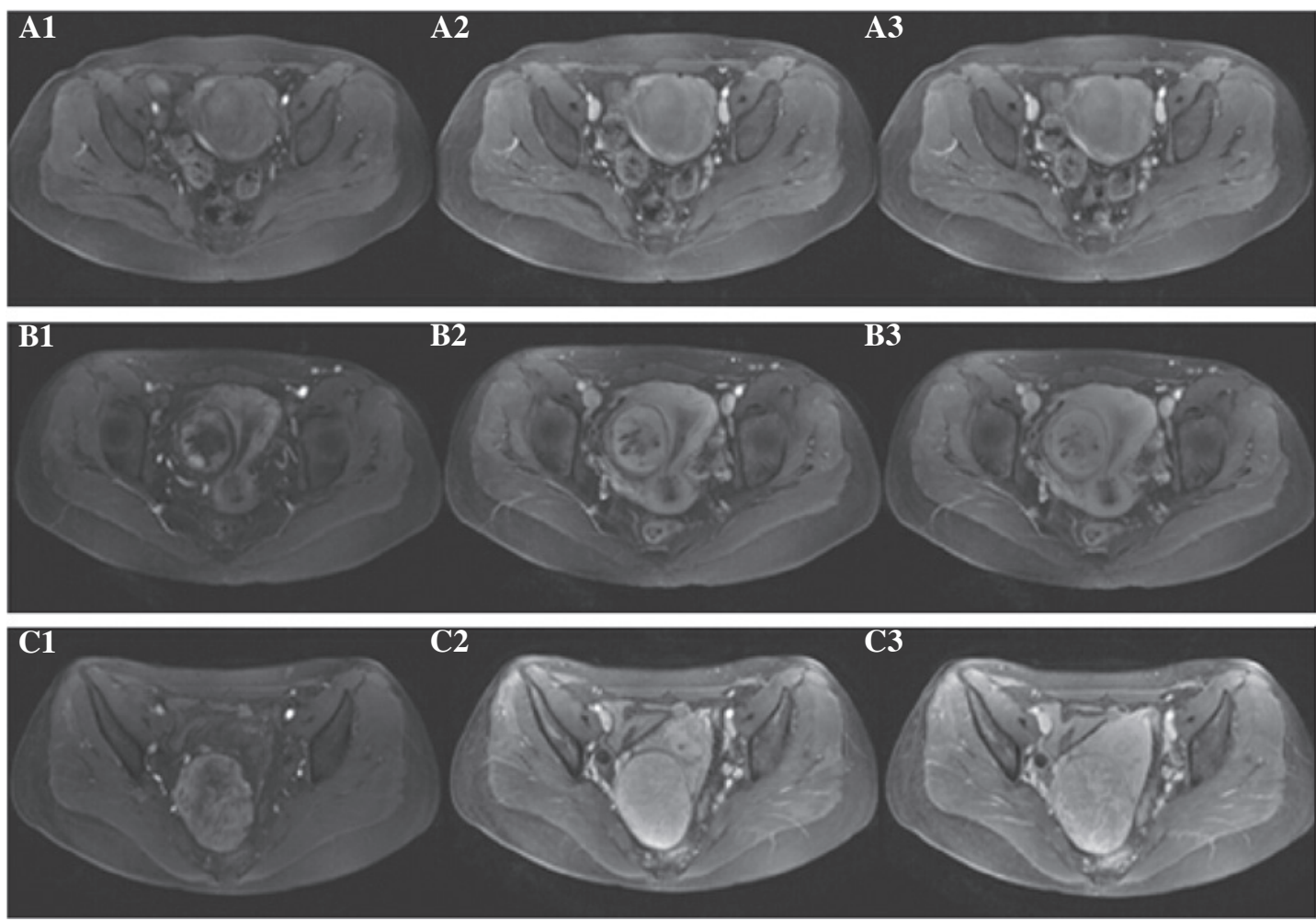

Figure 2. Dynamic contrast-enhanced Axial T1-weighted images of patients with hyperintense uterine fibroids corresponding to Fig. 1 prior to treatment. The images were acquired at (A) 20, (B) 40 and (C) $60 \mathrm{sec}$ following contrast injection. Fibroid shows (A) slight, (B) irregular and (C) progressive enhancement and began to exhibit a regular enhancement at $40 \mathrm{sec}$.
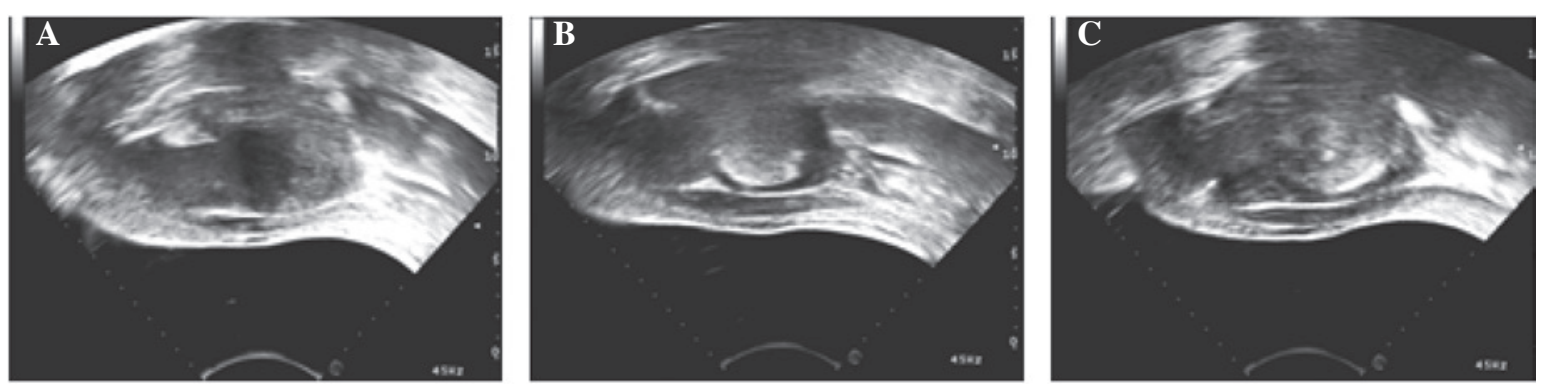

Figure 3. Real-time ultrasound images. (A) Uterine fibroid with hypoechoic and isoecho prior to treatment. (B) Gray scale changes are observed during treatment, partial hypoechoic and isoecho of fibroid translate into hyperecho. (C) Whole uterine fibroid is covered by hyperecho following treatment.

junctional zone and $\geq 1.5 \mathrm{~cm}$ from the endometrium in order to avoid damaging adjacent structures as a result of the heat produced by HIFU. The targeted area was treated layer by layer, and the thickness of one layer was $5 \mathrm{~mm}$. Once the area of gray-scale enhancement covered the majority of the lesion under ultrasound imaging, the physician discontinued the treatment (Fig. 3). All post-operative patients were observed for $2 \mathrm{~h}$ prior to discharge from the hospital. Oral prophylactic antibiotics were administered for the first three days following treatment. 
Table I. Characteristics of different types of uterine fibroids on dynamic contrast-enhanced MRI.

\begin{tabular}{|c|c|c|c|c|}
\hline Variable & $\begin{array}{c}\text { Irregular } \\
\text { enhancement }\end{array}$ & $\begin{array}{c}\text { Regular } \\
\text { enhancement }\end{array}$ & $\begin{array}{c}\text { Slight } \\
\text { enhancement }\end{array}$ & Total \\
\hline Fibroids, n (\%) & $44(33.6)$ & $48(36.6)$ & $39(29.8)$ & 131 \\
\hline Age, years & $38.0 \pm 6.0(22-49)$ & $36.7 \pm 6.7(24-49)$ & $37.2 \pm 7.0(23-50)$ & $37.2 \pm 6.5(22-50)$ \\
\hline BMI, $\mathrm{kg} / \mathrm{m}^{2}$ & $22.4 \pm 2.9(17.1-28.2)$ & $21.6 \pm 2.5(16.8-29.1)$ & $21.3 \pm 2.4(16.1-25.9)$ & $21.8 \pm 2.6(16.16-29.1)$ \\
\hline Uterine volume, $\mathrm{cm}^{3}$ & $262.7(186.9-378.7)$ & $233.3(178.2-366.8)$ & $295.7(188.9-389.3)$ & $267.6(182.8-386.0)$ \\
\hline $\begin{array}{l}\text { Fibroid location } \\
\text { IMF/SMF/SSF, n }\end{array}$ & $31 / 10 / 3$ & $34 / 11 / 3$ & $33 / 5 / 1$ & $98 / 26 / 7$ \\
\hline Fibroid volume, $\mathrm{cm}^{3}$ & $98.2^{\mathrm{a}}(53.0-150.1)$ & $69.7^{\mathrm{a}}(42.7-116.9)$ & $78.5(48.8-142.8)$ & $81.1(48.5-129.1)$ \\
\hline
\end{tabular}
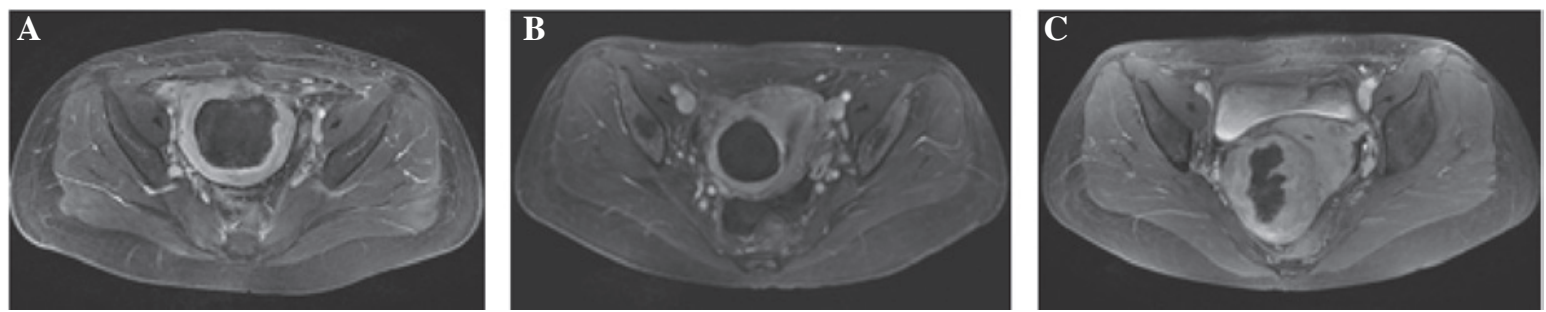

Figure 4. Contrast-enhanced axial T1-weighted images of patients with hyperintense uterine fibroids corresponding to Fig. 1 following treatment. The non-perfused volume (NPV) is visible inside uterine fibroids. (A and B) Fibroid with light enhancement has the highest NPV ratio. (C) Fibroid with regular enhancement has the lowest NPV ratio.

Assessment of the therapeutic effect. All the patients were asked to rapidly report any discomfort, which was carefully recorded during the procedure. The pain responses were scored according to the visual analog scale ( 0 -10 scale: 0 , no pain; 10 , most pain) $(14,15)$. The NPV was considered to be the fibroid necrosis volume (Fig. 4), and was evaluated within one month after the procedure by postoperative MRI, which were performed and evaluated by three experienced radiologists. The fibroid volume and the NPV were determined using the following equation: $\mathrm{V}=0.5233 \times \mathrm{D} 1 \times \mathrm{x}$ D2 x D3 (D1, longitudinal; D2, anteroposterior; D3, transverse).

Statistical analysis. All continuous variables were reported as the mean \pm standard deviation, and non-continuous variables were reported as the median and the interquartile range. SPSS software (version 19.0; IBM SPSS, Armonk, NY, USA) was used to analyze the results. One-way analysis of variance, the Mann-Whitney U test and $\chi^{2}$ test were used to analyze the baseline parameters, NPV ratio, pain scores, treatment duration, treatment efficiency, energy efficiency ratio and the adverse effects in patients with uterine fibroids with different types of enhancement on pretreatment dynamic contrast-enhanced MRI. $\mathrm{P}<0.05$ was considered to indicate a statistically significant difference.

\section{Results}

Patients and fibroids. A total of 131 patients met the inclusion criteria, and 131 fibroids were treated using USgHIFU. The mean age of the women was 37.2 \pm 6.5 years (range, $22-50$ years). The number of uterine fibroids with slight, irregular and regular enhancement was 39, 44 and 48, respectively. In addition, the mean fibroid diameter was $6.1 \mathrm{~cm}$ (range, $2.8-10 \mathrm{~cm}$ ) and the median volume of all of the fibroids was $81.1 \mathrm{~cm}^{3}$ (interquartile range, $48.5-129.1 \mathrm{~cm}^{3}$ ). Fibroids with a diameter of $>5 \mathrm{~cm}$ were primarily located in the wall of the uterus, followed by subserosal fibroids, which produced symptoms including pelvic pain, pressure, dysmenorrhea and urinary frequency. The majority of submucous fibroids were $<5 \mathrm{~cm}$ in diameter and resulted in menorrhagia and dysmenorrhea. The fibroids with regular enhancement exhibited a smaller volume compared with fibroids with irregular enhancement. Detailed information on the patients and the uterine fibroids is provided in Table I.

Post-procedure evaluation. The average acoustic sonication power was 403.0 $\pm 16.0 \mathrm{~W}$ (interquartile range, 354-499 W). The mean treatment time was defined as the period of time between the first and final sonication, which was $130 \mathrm{~min}$ (interquartile range, 68-173 $\mathrm{min}$ ). In addition, the mean sonication time was defined as the exposure time of the sonication emission, which was $28.9 \mathrm{~min}$ (interquartile range, 15.5-41.7 $\mathrm{min}$ ). The treatment times and the sonication times of the fibroids with regular enhancement were 149.0 min (interquartile range, 67.3-209.3 $\mathrm{min}$ ) and $37.5 \mathrm{~min}$ (interquartile range, 14.3-47.0 $\mathrm{min}$ ), respectively. These times were longer compared with those of the fibroids with slight or irregular enhancement $(\mathrm{P}<0.05)$. 
Table II. Treatment results of different types of uterine fibroids on dynamic contrast-enhanced MRI.

\begin{tabular}{|c|c|c|c|c|}
\hline Variable & $\begin{array}{c}\text { Irregular } \\
\text { enhancement }\end{array}$ & $\begin{array}{c}\text { Regular } \\
\text { enhancement }\end{array}$ & $\begin{array}{c}\text { Slight } \\
\text { enhancement }\end{array}$ & Total \\
\hline Power, W & $404.8 \pm 15.7(40-480)$ & $402.4 \pm 19.3(354-499)$ & $401.7 \pm 11.7(389-472)$ & $403.0 \pm 16.0(354-499)$ \\
\hline Treatment time, min & $130.5(68.5-153)$ & $149.0^{\mathrm{a}}(67.3-209.3)$ & $105.0^{\mathrm{a}}(72.0-143.0)$ & $130(68-173)$ \\
\hline Sonication time, $\min$ & $27.7^{\mathrm{b}}(15.7-41.7)$ & $37.5^{\mathrm{b}, \mathrm{c}}(14.3-47.0)$ & $26.1^{\mathrm{c}}(17.1-37.9)$ & $28.9(15.5-41.7)$ \\
\hline Treatment efficiency, $\mathrm{mm}^{3} / \mathrm{s}$ & $40.5^{\mathrm{d}}(23.1-56.6)$ & $18.8^{\mathrm{d}, \mathrm{e}}(9.3-40.8)$ & $38.8^{\mathrm{e}}(29.7-76.4)$ & $34.3(17.4-56.8)$ \\
\hline $\begin{array}{l}\text { Energy efficiency } \\
\text { ratio, } \mathrm{J} / \mathrm{mm}^{3}\end{array}$ & $9.5^{f}(6.7-16.2)$ & $19.9^{\mathrm{d}, \mathrm{f}}(8.7-42.2)$ & $10.5^{\mathrm{d}}(5.3-13.4)$ & $11.4(7.0-21.8)$ \\
\hline NPV ratio, $\%$ & $\begin{array}{l}70.6 \pm 17.4^{\mathrm{g}} \\
(33.3-100)\end{array}$ & $\begin{array}{c}57.1 \pm 26.8^{\mathrm{e}, \mathrm{g}} \\
(0-99.6)\end{array}$ & $\begin{array}{c}84.7 \pm 17.1^{\mathrm{e}, \mathrm{g}} \\
(47.5-100)\end{array}$ & $\begin{array}{c}68.5 \pm 24.1 \\
(0.0-100)\end{array}$ \\
\hline
\end{tabular}

Continuous variables presented as the mean \pm standard deviation and non-continuous variables presented as the median with the interquartile range. ${ }^{\mathrm{P}} \mathrm{P}=0.002,{ }^{\mathrm{b}} \mathrm{P}=0.032,{ }^{\mathrm{c}} \mathrm{P}=0.028,{ }^{\mathrm{d}} \mathrm{P}=0.001,{ }^{\mathrm{e}} \mathrm{P}<0.001,{ }^{\mathrm{f}} \mathrm{P}=0.006,{ }^{\mathrm{g}} \mathrm{P}=0.009$. MRI, magnetic resonance imaging; NPV, non-perfused volume

Table III. Incidence of various pain types and pain score during treatment with USgHIFU.

\begin{tabular}{|c|c|c|c|c|}
\hline Variable & $\begin{array}{c}\text { Irregular } \\
\text { enhancement }\end{array}$ & $\begin{array}{c}\text { Regular } \\
\text { enhancement }\end{array}$ & $\begin{array}{c}\text { Slight } \\
\text { enhancement }\end{array}$ & Total \\
\hline \multicolumn{5}{|l|}{ Pain type, \% (n) } \\
\hline Radiating & $36.4(16)$ & $35.4(17)$ & $28.2(11)$ & $33.6(44)$ \\
\hline Skin burning sensation & $59.1(26)$ & $64.6(31)$ & $46.2(18)$ & $57.3(75)$ \\
\hline Lower abdominal & $72.7(32)$ & $68.8(33)$ & $69.2(27)$ & $70.2(92)$ \\
\hline Hip & $45.5(20)$ & $50.0(24)$ & $38.5(18)$ & $47.3(62)$ \\
\hline Sacrococcygeal & $63.6(28)$ & $62.7(30)$ & $61.5(24)$ & $62.6(82)$ \\
\hline Overall pain, \% (n) & $90.9(40)$ & $95.8(46)$ & $89.7(35)$ & $92.3(121)$ \\
\hline Pain score & $3.2 \pm 0.9^{\mathrm{a}}(1-5)$ & $3.7 \pm 0.9^{\mathrm{ab}}(2-5)$ & $3.0 \pm 0.9^{\mathrm{b}}(2-4)$ & $3.3 \pm 0.9(1-5)$ \\
\hline
\end{tabular}

${ }^{\mathrm{a}} \mathrm{P}=0.012,{ }^{\mathrm{b}} \mathrm{P}=0.001$. USgHIFU, ultrasound-guided high-intensity focused ultrasound.

The treatment efficiency was defined as the volume of fibroid tissue $\left(\mathrm{mm}^{3}\right)$ that was ablated during a one-second sonication emission. The energy efficiency ratio was defined as the joules of energy required when ablating $1 \mathrm{~mm}^{3}$ fibroid tissue. The mean NPV ratio of all of the uterine fibroids was $68.5 \pm 24.1 \%$ (range, $0-100 \%$ ). Furthermore, the median treatment efficiency and the energy efficiency ratio were $34.3 \mathrm{~mm}^{3} / \mathrm{s}$ (interquartile range, $17.4-56.8 \mathrm{~mm}^{3} / \mathrm{s}$ ) and $11.4 \mathrm{~J} / \mathrm{mm}^{3}$ (interquartile range, $7.0-21.8 \mathrm{~J} / \mathrm{mm}^{3}$ ), respectively. The NPV ratio, the treatment efficiency and the energy efficiency ratio of the uterine fibroids with slight, irregular or regular enhancement are described in Table II. Fibroids with regular enhancement were associated with a lower NPV ratio and lower treatment efficiency compared with those of fibroids with irregular or slight enhancement $(\mathrm{P}<0.05)$. However, the energy efficiency ratio was the highest for the fibroids with regular enhancement $(\mathrm{P}<0.05)$.

Adverse events. All adverse events were well-tolerated, and none of the patients presented serious adverse events that corresponded with the unified standardized Society of Interventional Radiology grading system (16) during treatment with USgHIFU. The most common adverse events were lower abdominal pain (70.2\%), sacrococcygeal pain (62.6\%), a skin burning sensation $(57.3 \%)$, hip pain $(45.0 \%)$ and radiating pain in the lower limbs (33.6\%). The differences in the incidence of pain were not statistically significant between patients who had fibroids with slight, irregular or regular enhancement $(\mathrm{P}>0.05)$. The mean pain score was $3.3 \pm 0.9$ points (range, $1-5$ points) for all patients. In addition, the mean pain score of patients that had fibroids with regular enhancement was 3.7 \pm 0.9 points (range, 2-5 points), which was higher compared with that of patients who had fibroids with slight or irregular enhancement $(\mathrm{P}<0.05$; Table III).

\section{Discussion}

Lénárd et al (8) and Funaki et al (9) have observed that the NPV ratio of hyperintense uterine fibroids is reduced compared with that of hypointense and isointense fibroids; therefore, HIFU may not be an appropriate treatment method for hyperintense fibroids. However, the results of the present study indicated that the majority of hyperintense uterine fibroids may be eligible for HIFU ablation, based on the technical success of the present procedures. Fibroids with regular enhancement in the arterial phase of dynamic contrast-enhanced MRI were 
associated with the lowest NPV ratio and the lowest treatment efficiency, but with the highest energy efficiency ratio and risk of severe adverse effects.

An increase in the NPV ratio was associated with an increase in the fibroid volume reduction ratio and symptomatic relief (2). In the present study, USgHIFU ablation technology generated a mean NPV ratio of $68.5 \%$ among all hyperintense fibroids; however, fibroids with regular enhancement were associated with the lowest ratio (57.1\%). Stewart et al (2) suggested that when the NPV ratio of fibroids is $<36.6 \%$, the probability of additional treatment may be $>10 \%$, which indicates a worse long-term curative effect for patients that have fibroids with regular enhancement. These fibroids may not undergo a significant reduction in volume.

According to the present results, fibroids with regular enhancement may be associated with a lower treatment efficiency and a higher energy efficiency ratio compared with fibroids with slight or irregular enhancement. In the present study, the fibroids with regular enhancement had a smaller volume compared with the other two groups of fibroids; however, these fibroids required longer treatment and sonication times.

It is hypothesized that the blood flow of fibroids may be a crucial factor influencing ablation. Dynamic contrast-enhanced MRI is performed using a T1-weighted gradient-echo sequence after pump injection of $0.1 \mathrm{mmol} / \mathrm{kg}$ gadolinium, and is able to indicate the perfusion and permeability of a tumor and is used to distinguish malignant lesions from benign lesions, in addition to distinguishing degenerative lesions from 'ordinary' lesions. According to a number of studies $(12,17,18)$, dynamic contrast-enhanced MRI may be used to distinguish between degenerative fibroids and cellular fibroids, which both manifest as hyperintense under T2-weighted MRI. Cellular fibroids are copiously supplied by vessels and manifest with regular enhancement on dynamic contrast-enhanced MRI scans. As degenerative fibroids have reduced blood supply, they manifest with slight or irregular enhancement (12).

Fibroids with rich blood flow or significant cellularity may have been resistant to HIFU, as the energy could not be easily deposited $(12,17,18)$. By contrast, degenerative fibroids are easily ablated by HIFU as they contain limited cellularity and diminished blood flow. Therefore, the fibroids with regular enhancement had a smaller volume compared with the other fibroids prior to treatment, as ischemia and degeneration occur most frequently in larger uterine fibroids. The vessel density in the fibroids and the enhancement degree were positively correlated at $60 \mathrm{sec}$ after the injection of gadolinium (19). The fibroids with abundant vessels were well-enhanced, while the fibroids with severe degeneration were poorly enhanced (20). Furthermore, the images within $60 \mathrm{sec}$ corresponded with the arterial phase and were representative of arterial blood flow (19). The images in the later stages indicated the effect of contrast materials in the extravascular space rather than in the endovascular space (21). Therefore, dynamic contrast-enhanced MRI in the arterial phase exhibited improved clinical value in determining the blood flow within the fibroids and in predicting the treatment effect of USgHIFU ablation for the treatment of uterine fibroids. The results of the present study demonstrated that hyperintense fibroids with slight or irregular enhancement on dynamic contrast-enhanced MRI scans may be successfully treated using USgHIFU.

Regarding the occurrence of adverse effects, Hindley et al (1) reported that $82 \%$ of patients suffered varying degrees of pain during treatment. In the present study, the incidence of pain, including radiating pain, sacrococcygeal pain, hip pain and lower abdominal pain, was $100 \%$. The patients that had fibroids with regular enhancement exhibited higher pain scores compared with the other patients, which may be explained by the longer treatment times and the greater sonication energy that were required for the treatment of hyperintense fibroids $(22,23)$. Among the various adverse events, a higher incidence of radiating pain and skin burning sensations was observed in the patients that had fibroids with regular enhancement, which may increase the risk of neurotoxic effects in their legs and skin burning sensations; however, there were no statistically significant differences. Therefore, fibroids with regular enhancement may increase the risk of severe adverse effects in patients.

A number of limitations exist in the current study, which was a feasibility study of USgHIFU ablation for the treatment of hyperintense uterine fibroids. Randomized controlled studies are required to confirm the long-term safety and efficacy of this procedure. Further quantification of the enhancement degree of T2 hyperintense uterine fibroids based on dynamic contrast-enhanced MRI scans may improve the accuracy of such studies. In addition, further studies comparing HIFU with other available methods for the treatment of hyperintense fibroids may lead to improved treatment options in the future.

To conclude, the results of the present study suggest that hyperintense uterine fibroids with slight and irregular enhancement in the arterial phase of dynamic contrast-enhanced MRI after an injection of gadolinium are suitable for USgHIFU treatment. However, fibroids with regular enhancement were associated with the worst treatment efficacy and safety.

\section{Acknowledgements}

This study was supported by the National Basic Research Program of China (grant nos. 2011CB707900 and 2012CB722402), the National Key Technology R\&D Program (grant no. 2011BAI14B01), and the National Natural Science Fund by the Chinese National Science Foundation (grant nos. 81127901, 31000435 and 11274404).

\section{References}

1. Hindley J, Gedroyc WM, Regan L, Stewart E, Tempany C, Hynyen K, Mcdannold N, Inbar Y, Itzchak Y, Rabinovici J, et al: MRI guidance of focused ultrasound therapy of uterine fibroids: Early results. AJR Am J Roentgenol 183: 1713-1719, 2004.

2. Stewart EA, Gostout B, Rabinovici J, Kim HS, Regan L and Tempany CM: Sustained relief of leiomyoma symptoms by using focused ultrasound surgery. Obstet Gynecol 110: 279-287, 2007.

3. Edozien LC: Costing magnetic resonance-guided focused ultrasound surgery, a new treatment for symptomatic fibroids. BJOG 115: 1321, 2008.

4. Stewart EA, Rabinovici J, Tempany CM, Inbar Y, Regan L, Gostout B, Hesley G, Kim HS, Hengst S and Gedroyc WM: Clinical outcomes of focused ultrasound surgery for the treatment of uterine fibroids. Fertil Steril 85: 22-29, 2006. 
5. Fennessy FM, Tempany CM, McDannold NJ, So MJ, Hesley G, Gostout B, Kim HS, Holland GA, Sarti DA, Hynynen K, et al: Uterine leiomyomas: MR imaging-guided focused ultrasound surgery - results of different treatment protocols. Radiology 243 : 885-893, 2007.

6. LeBlang SD, Hoctor K and Steinberg FL: Leiomyoma shrinkage after MRI-guided focused ultrasound treatment: Report of 80 patients. AJR Am J Roentgenol 194: 274-280, 2010.

7. Funaki K, Fukunishi H, Funaki T, Sawada K, Kaji Y and Maruo T: Magnetic resonance-guided focused ultrasound surgery for uterine fibroids: Relationship between the therapeutic effects and signal intensity of preexisting T2-weighted magnetic resonance images. Am J Obstet Gynecol 196: 184.el-e6, 2007.

8. Lénárd ZM, McDannold NJ, Fennessy FM, Stewart EA, Jolesz FA, Hynynen K and Tempany CM: Uterine leiomyomas: MR imaging-guided focused ultrasound surgery - imaging predictors of success. Radiology 249: 187-194, 2008.

9. Funaki K, Fukunishi H and Sawada K: Clinical outcomes of magnetic resonance-guided focused ultrasound surgery for uterine myomas: 24-month follow-up. Ultrasound Obstet Gynecol 34: 584-589, 2009.

10. Gorny KR, Woodrum DA, Brown DL, Henrichsen TL, Weaver AL, Amrami KK, Hangiandreou NJ, Edmonson HA, Bouwsma EV, Stewart EA, et al: Magnetic resonance-guided focused ultrasound of uterine leiomyomas: review of a 12 -month outcome of 130 clinical patients. J Vasc Interv Radiol 22: 857-864, 2011.

11. Yoon SW, Lee C, Kim KA and Kim SH: Contrast-enhanced dynamic MR imaging of uterine fibroids as a potential predictor of patient eligibility for MR guided focused ultrasound (MRgFUS) treatment for symptomatic uterine fibroids. Obstet Gynecol Int 2010: 834275, 2010.

12. Yamashita $\mathrm{Y}$, Torashima $\mathrm{M}$, Takahashi $\mathrm{M}$, Tanaka $\mathrm{N}$, Katabuchi H, Miyazaki K, Ito M and Okamura H: Hyperintense uterine leiomyoma at T2-weighted MR imaging: Differentiation with dynamic enhanced MR imaging and clinical implications. Radiology 189: 721-725, 1993.

13. Thomassin-Naggara I, Daraï E, Nassar-Slaba J, Cortez A, Marsault C and Bazot M: Value of dynamic enhanced magnetic resonance imaging for distinguishing between ovarian fibroma and subserous uterine leiomyoma. J Comput Assist Tomogr 31: 236-242, 2007.

14. McCaffery M: Using the 0 -to-10 pain rating scale. Am J Nurs 101: 81-82, 2001.
15. Farrar JT, Pritchett YL, Robinson M, Prakash A and Chappell A The clinical importance of changes in the 0 to 10 numeric rating scale for worst, least, and average pain intensity: Analyses of data from clinical trials of duloxetine in pain disorders. J Pain 11: $109-118,2010$.

16. Goldberg SN, Grassi CJ, Cardella JF, Charboneau JW, Dodd GD 3rd, Dupuy DE, Gervais DA, Gillams AR, Kane RA, Lee FT Jr, et al; Society of Interventional Radiology Technology Assessment Committee and the International Working Group on Image-guided Tumor Ablation: Image-guided tumor ablation: Standardization of terminology and reporting criteria. J Vasc Interv Radiol 20 (Suppl): S377-S390, 2009.

17. Oguchi O, Mori A, Kobayashi Y, Horiuchi A, Nikaido T and Fujii S: Prediction of histopathologic features and proliferative activity of uterine leiomyoma by magnetic resonance imaging prior to $\mathrm{GnRH}$ analogue therapy: correlation between T2-weighted images and effect of GnRH analogue. J Obstet Gynaecol (Tokyo 1995) 21: 107-117, 1995.

18. Murase E, Siegelman ES, Outwater EK, Perez-Jaffe LA and Tureck RW: Uterine leiomyomas: Histopathologic features, MR imaging findings, differential diagnosis, and treatment. Radiographics 19: 1179-1197, 1999.

19. Shimada K, Ohashi I, Kasahara I, Watanabe H, Ohta S, Miyasaka N, Itoh E and Shibuya H: Differentiation between completely hyalinized uterine leiomyomas and ordinary leiomyomas: three-phase dynamic magnetic resonance imaging (MRI) vs. diffusion-weighted MRI with very small b-factors. J Magn Reson Imaging 20: 97-104, 2004.

20. Shimada K, Ohashi I, Kasahara I, Miyasaka N and Shibuya H: Triple-phase dynamic MRI of intratumoral vessel density and hyalinization grade in uterine leiomyomas. AJR Am J Roentgenol 182: 1043-1050, 2004.

21. Choyke PL, Dwyer AJ and Knopp MV: Functional tumor imaging with dynamic contrast-enhanced magnetic resonance imaging. J Magn Reson Imaging 17: 509-520, 2003.

22. $\mathrm{Yu} \mathrm{T}$ and Luo J: Adverse events of extracorporeal ultrasound-guided high intensity focused ultrasound therapy. PLoS One 6: e26110, 2011.

23. Yang WW, Zhu BR, Li J, Xia WX, Liu Y, Gai LH, Zhou JM and Sun JF: Analysis of complications of high intensity focused ultrasound in treatment of uterine leiomyoma. Zhong $\mathrm{Hua} \mathrm{Fu}$ Chan Ke Za Zhi 45: 913-916, 2010 (In Chinese). 